\title{
HoCa Home Care Multi-Agent Architecture
}

\author{
Juan A. Fraile ${ }^{1}$, Javier Bajo ${ }^{1}$, Belén Pérez Lancho ${ }^{2}$, and Eladio Sanz ${ }^{2}$ \\ 1 Universidad Pontificia de Salamanca, Compañía 5, 37002, Salamanca, Spain, \\ \{jafraileni, jbajope\}@upsa.es \\ 2 Departamento de Informática y Automática, Universidad de Salamanca, Plaza \\ de la Merced s/n, 37008, Salamanca, Spain, \{lancho, esanz\}@usal.es
}

Summary. This paper presents a Hybrid Multi-Agent Architecture for the control and supervision of dependent environments, based on an Ambient Intelligence model. HoCa architecture incorporates a management system of alerts based on SMS and MMS technologies, and an automated identification, localization, and movement control system based on Java Card and RFID technologies. HoCa is independent from the programming language and operating system in that it is executable. The core of the architecture is formed by both deliberative agents and reactive agents that interact to offer efficient services. The architecture has been tested in a real environment and the results obtained are presented in this paper.

Key words: Dependent environments, Ambient Intelligence, Multiagent Systems, Home Care

\section{Introduction}

There is currently considerable growth in the development of automation technologies, such as home automation and Ambient Intelligence (AmI). One of their principal objectives is to look after the user's well-being and obtain a more friendly, rational, productive, sustainable and secure relationship for users within their environments. Several architectures based on agent utilization have emerged thanks to the appearance of intelligent spaces and the integration of devices that are programmable via computer networks [11]. These have stimulated the development of ubiquitous computation, which is the most promising technological approximation for resolving the challenge of developing strategies that allow the early detection and prevention of problems in an automated environment.

The main objective of this paper is to define a hybrid Multi-Agent Architecture for the control and the supervision of open environments. It involves developing an architecture that allows automated identification, localization, 
alarms management and control of movement. The users who utilize the system in which this architecture is applied will be able to gain wireless access to all the information that they need to perform their work. The novel innovation at the core of the architecture is a real time communication protocol that allows secure and rapid communication between the reactive agents and the system sensors. These reactive agents, whose response time is critical, are influenced by deliberative BDI agents, which are located inside the platform given that a very fluid communication already exists between them. Additionally, the architecture manages an alert or alarm system across the agents' platform specially designed to work with mobile devices. The alert system contains different levels of urgency. The alert level is determined by the deliberative agent who, depending on the alert level, then emits the alert to either a reactive agent or a deliberative agent.

The paper is organized as follows: The second section presents the problem that prompted this work. The third section presents the proposed architecture, and the fourth section gives the results and conclusions obtained after applying the proposed architecture to a real case in an environment of dependence.

\section{General Description of the Problem}

The use of intelligent agents is an essential component for analyzing information on distributed sensors [12] [14]. These agents must be capable of both independent reasoning and joint analysis of complex situations in order to be able to achieve a high level of interaction with humans [3]. Although multiagent systems already exist and are capable of gathering information within a given environment in order to provide medical care [9] [5], there is still much work to be done. It is necessary to continue developing systems and technology that focus on the improvement of services in general. After the development of the internet there has been continual progress in new wireless communication networks and mobile devices such as mobile telephones and PDAs. This technology can help to construct more efficient distributed systems capable of addressing new problems [6].

Hybrid architectures try to combine deliberative and reactive aspects, by combining reactive and deliberative modules [5]. The reactive modules are in charge of processing stimuli that do not need deliberation, whereas the deliberative modules determine which actions to take in order to satisfy the local and cooperative aims of the agents. The aim of modern architectures like Service Oriented Architecture (SOA) is to be able to interact among different systems by distributing resources or services without needing to consider which system they are designed for. An alternative to these architectures are the multi-agent systems, which can help to distribute resources and to reduce the centralization of tasks. Unfortunately the complexity of designing multiagent architecture is great since there are not tools to either help programme needs or develop agents. 
Multi-agent systems combine aspects of both classic and modern architectures. The integration of multi-agent systems with SOA and web services has been recently investigated [1]. Some investigators focus on the communication among these models, whereas others focus on the integration of distributed services, especially web services, in the agents' structure [3] [10] [13].

These works provide a good base for the development of multi-agent systems. Because the majority of them are in the development stage, their full potential in a real environment is not known. HoCa has been implemented in a real environment and not only does it provide communication and integration among distributed agents, services and applications, but it also provides a new method for facilitating the development of multi-agent systems, thus allowing the agents and systems to function as services.

HoCa implements an alert and alarm system across the agent's platform, specially designed to be used by mobile devices. The platform agents manage this service and determine the level of alert at every moment so that they can decide who will receive the alert and when. In order to identify each user, HoCa implements a system based on Java Card [15] and RFID (Radio Frequency IDentification) microchip technology in which there will be a series of distributed sensors that provide the necessary services to the user.

\section{Proposed Architecture}

The HoCa model architecture uses a series of components to offer a solution that includes all levels of service for various systems. It accomplishes this by incorporating intelligent agents, identification and localization technology, wireless networks and mobile devices. Additionally, it provides access mechanisms to multi-agent system services, through mobile devices, such as mobiles phones or PDAs. Access is provided via wi-fi wireless networks, a notification and alarm management module based on SMS and MMS technologies, and user identification and localization system based on Java Card and RFID technologies. This system is dynamic, flexible, robust and very adaptable to changes of context.

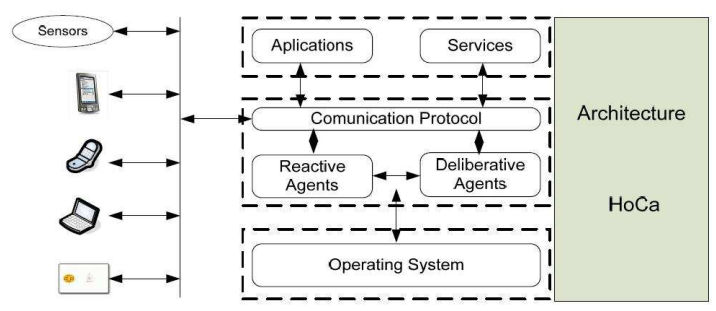

Fig. 1. HoCa Framework 
HoCa architecture describes four basic blocks that can be seen in Figure 1: Applications, Services, Agents Platform and Communication Protocol. These blocks constitute the whole functionality of the architecture.

\subsection{Agents Platform in $\mathrm{HoCa}$}

This platform is the core of the architecture and integrates two types of agents, each of which behaves differently for specific tasks, as shown in Figure 2.

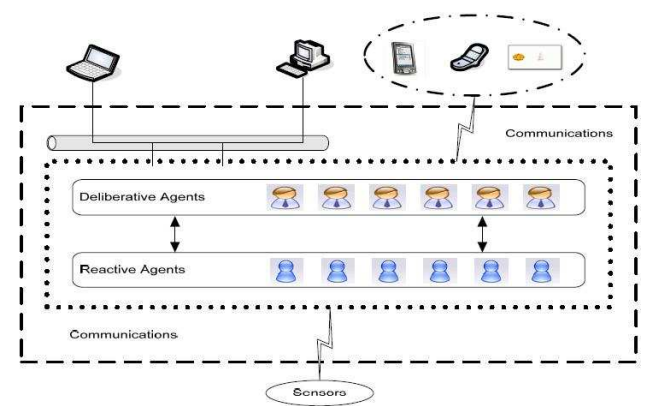

Fig. 2. Agents platform structure in the HoCa Architecture

The first group of agents is made up of deliberative BDI agents, which are in charge of the management and coordination of all system applications and services. These agents are able to modify their behaviour according to the preferences and knowledge acquired in previous experiences, thus making them capable of choosing the best solution. Deliberative agents constantly deal with information and knowledge. Because they can be executed on mobile devices, they are always available and they provide ubiquitous access for the users. There are different kinds of agents in the architecture, each one with specific roles, capabilities and characteristics. This fact facilitates the flexibility of the architecture to incorporate new agents. However, there are pre-defined agents which provide the basic functionalities of the architecture:

- CoAp Agent: This agent is responsible for all communications between applications and the platform. Manages the incoming requests from the applications to be processed by services. It also manages responses from services to applications. CoAp Agent is always on "listening mode". Applications send XML messages to the agent requesting for a service, then the agent creates a new thread to start communication using sockets.

- CoSe Agent: It is responsible for all communications between services and the platform. The functionalities are similar to CommApp Agent but backwards. This agent is always on "listening mode" waiting for responses of services. Manager Agent indicates CommServ Agent the service that must be invoked. Then, CommServ Agent creates a new thread with its respective socket and sends an XML message to the service. 
- Directory Agent. Manages the list of services that can be used by the system. For security reasons, the list of services is static and can only be modified manually, however services can be added, erased or modified dynamically. The list contains the information of all trusted available services.

- Supervisor Agent. This agent supervises the correct functioning of the agents in the system. Supervisor Agent verifies periodically the status of all agents registered in the architecture by means of sending ping messages. If there is no response, the agent kills the agent and creates another instance of that agent.

- Security Agent. This agent analyzes the structure and syntax of all incoming and outgoing XML messages. If a message is not correct, the Security Agent informs the corresponding agent that the message cannot be delivered.

- Manager Agent. Decides which agent must be called taking into account the users preferences. Users can explicitly invoke a service, or can let the Manager Agent decide which service is better to accomplish the requested task. If there are several services that can resolve the task requested by an application, the agent selects the optimal choice.

- Interface Agent. This kind of agent has been designed to be embedded in users' applications. Interface agents communicate directly with the agents in HoCa so there is no need to employ the communication protocol, but FIPA ACL specification. The requests are sent directly to the Security Agent, which analyzes the requests and sends them to the Manager Agent.

The second group is made up of reactive agents. Most of the research conducted within the field of multi-agent systems focuses on designing architectures that incorporate complicated negotiation schemes as well as high level task resolution, but don't focus on temporal restrictions. In general, the multi-agent architectures assume a reliable channel of communication and, while some establish deadlines for the interaction processes, they don't provide solutions for limiting the time the system may take to react to events.

It is possible to define a real-time agent as an agent with temporal restrictions for some of its responsibilities or tasks [11]. From this definition, we can define a real-time multi-agent system (Real Time Multi-Agent System, RTMAS) as a multi-agent system in which at least one of the agents is a real-time agent. The use of RT-MAS makes sense within an environment of critical temporal restrictions, where the system can be controlled by autonomous agents that need to communicate among themselves in order to improve the degree of system task completion. In this kind of environments every agent requires autonomy as well as certain cooperation skills to achieve a common goal.

\subsection{HoCa Communication Protocol}

Communication protocol allows applications, services and sensors to be connected directly to the platform agents. The protocol presented in this work is 
open and independent of programming languages. It is based on the SOAP standard and allows messages to be exchanged between applications and services as shown in Figure 3.

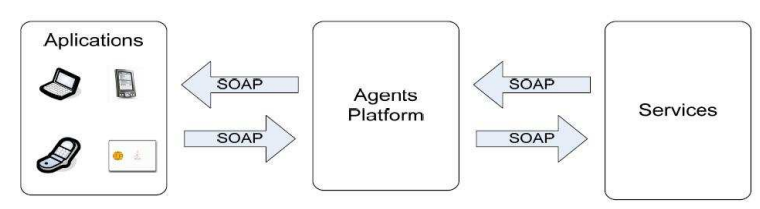

Fig. 3. Communication using SOAP messages in HoCa

However, interaction with environmental sensors requires Real-time Transport Protocol (RTP) [4] which provides transport functions that are adapted for applications that need to transmit real-time data such as audio, video or simulation data, over multicast or unicast network services. The RTCP protocol is added to RTP, allowing a scaleable form of data supervision. Both RTP and RTCP are designed to work independently from the transport and lower network services. They are in charge of transporting data with realtime characteristics, and of supervising the quality of service, managing the information for all the entities taking part in the current session.

The communications between agents within the platforms follows the FIPA ACL (Agent Communication Language) standard. This way, the applications can use the platform to communicate directly with the agents.

\subsection{Location and Identification System in HoCa}

This system incorporates Java Card [15] and RFID [7] technologies. The primary purpose of the system is to convey the identity of an object or person, as with a unique serial number, using radio waves. Java Card is a technology that permits small Java applications (applets) to be run safely in microchip smart cards and similar embedded devices. Java Card gives the user the ability to program applications that can be run off a card so that it has a practical function in a specific application domain. The main features of Java Card are portability and security; it is described in ISO 7816. The data are stored in the application and the Java Card applications are executed in an isolated environment, separate from the operating system and from computer that reads the card. The most commonly used algorithms, such as DES, 3DES, AES, and RSA, are cryptographically implemented in Java Card. Other services such as electronic signature or key generation are also supported.

RFID technology is grouped into the so-called automatic identification technologies. But RFID provides more information than other auto-identification technologies, speeds up processes without losing reliability, and requires no human intervention. 
The combination of these two technologies allows us to both identify the user or identifiable element, and to locate it, by means of sensors and actuators, within the environment, at which time we can act on it and provide services. The microchip, which contains the identification data of the object to which it is adhered, generates a radio frequency signal with this data. The signal can be picked up by an RFID reader, which is responsible for reading the information and sending it, in digital format, to the specific application.

\subsection{Using HoCa to development a Multi-Agent System for dependent environment}

The alert system is integrated into the HoCa architecture and uses mobile technology to inform users about alerts, warnings and information specific to the daily routine of the application environment. This is a very configurable system that allows users to select the type of information they are interested, and to receive it immediately on their mobile phone or PDA. It places the information to be sent into information categories. The users determine the information they are interested in. The system automatically sends the information to each of the users as soon as it is available.

\section{Proposed Architecture}

Ambient Intelligence based systems aim to improve people quality of life, offering more efficient and easy to use services and communication tools to interact with other people, systems and environments. One of the most benefited segments of population with the development of these systems is elderly and dependent people. Agents and multi-agent systems in dependency environments are becoming a reality, especially on health care. Most agents-based applications are related to the use of this technology in patients monitoring, treatment supervision and data mining.

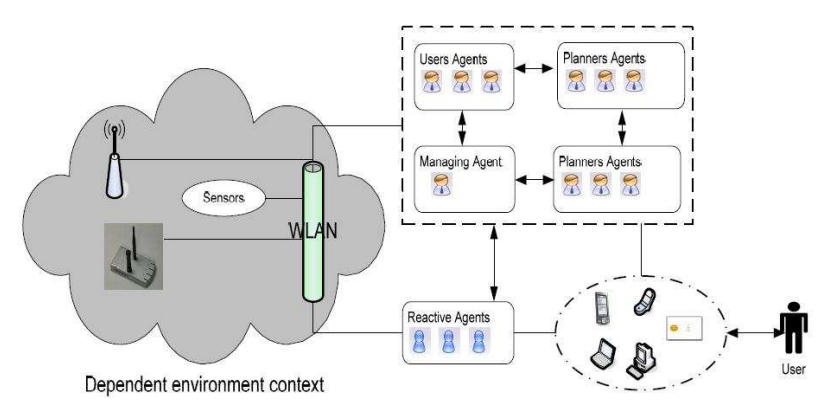

Fig. 4. HoCa structure in a dependent environment 
HoCa has been employed to develop a multi-agent system aimed to enhance assistance and care for low dependence patients at their homes. Main functionalities in the system include reasoning, planning mechanisms, management alerts and responses in execution time offered to certain stimuli, as shown in Figure 4. These functionalities allow the system the use of several context-aware technologies to acquire information from users and their environment. Among the technologies used are mobile systems for alerts service managing across PDA and mobile phones, Java Card elements for identification and presence detectors and access control.

Each agent in the system has its own functionalities. If an agent needs to develop a task in collaboration with other agent a request form is send. There are priority tasks that a set of agents can perform. This ensures that the priority tasks are always available. There are four types of agents:

- User Agents manage user personal data and their behavior. They are responsible through the system, identify and locate implemented by the architecture. They determine the status of the user and offering services in the environment as a correct temperature, automatic lighting, access blocking or opening, etc.

- SuperUser Agent runs on mobile devices and inserts new tasks into the Manager Agent to be processed by a reasoning mechanism. It also needs to interact with the User Agents to impose new tasks and receive periodic reports, and with the ScheduleUser Agents to ascertain plans' evolution.

- SheduleUser Agent schedules the users' daily activities obtaining dynamic plans depending on the tasks needed for each user. It manages scheduledusers profiles, tasks, available time and resources. Every agent generates personalized plans depending on the scheduled-user profile.

- Manager Agent runs on a Workstation and plays two roles: the security role that monitors the users and the manager role that handle the databases and the tasks assignment. It must provide security for the users and ensure the tasks assignments are efficient.

On the other hand there are a number of reactive agents that work in collaboration with the deliberative agents. These agents are in change of control devices interacting with sensors (access points, lights, temperature, alarms detection, etc.). They receive information, monitor environment services and also check the devices status connected to the system. All information is treated by the reactive agent and it is sent to the manager agent to be processed.

\section{Results and Conclusions}

HoCa has been used to develop a multi-agent system for monitoring dependent patients at home. The main features of this system include reasoning and planning mechanisms, and alert and response management. Most of these responses are reactions in real time to certain stimuli, and represent the abilities 
that the reactive agents have in the HoCa architecture based platform. To offer all these features the system uses various technologies and acquires information from users and the surrounding environment. Some of the technologies used to test the system include mobile technology for managing service alerts through PDAs and mobile phones, and Java Card technology for identification and access control.

One of the main contributions of the HoCa architecture is the alert system. We implemented several test cases to evaluate the management of alerts integrated into the system. This allowed us to determine the response time for warnings generated by the users, for which the results were very satisfactory, with response times shorter than those obtained prior to the implementation of HoCa. The system studies the information collected, and applies a reasoning process which allows alerts to be automatically generated. For these alerts, the system does not only take response time into account, but also the time elapsed between alerts, and the user's profile and reliability, in order to generalize reactions to common situations. The results show that $\mathrm{HoCa}$ fits perfectly within complex systems by correctly exploiting services and planning mechanisms.

Table 1. Comparison between the HoCa and the ALZ-MAS architectures.

\begin{tabular}{lll}
\hline Factor & HoCa ALZ-MAS \\
\hline Average response time to incidents (min.) & 8 & 14 \\
Assisted incidents & 12 & 17 \\
Average number of daily planned tasks & 12 & 10 \\
Average number of daily services completed & 46 & 32 \\
Time employed to attend and alert (min.) & 75 & 90 \\
\hline
\end{tabular}

Table 1 presents the results obtained after comparing the HoCa architecture to the previously developed ALZ-MAS architecture [6] in a case study on medical care for patients at home. The ALZ-MAS architecture allows the monitoring of patients in geriatric residences, but home care is carried out through traditional methods. The case study presented in this work consisted of analysing the functioning of both architectures in a test environment. The HoCa architecture was implemented in the home of 5 patients and was tested for 30 days. The results were very promising. The data shown in Table 1are the results obtained from the test cases. They show that the alert system improved the communication between the user and the dependent care services providers, whose work performance improved, allowing them to avoid unnecessary movement such as travels and visits simply oriented to control or supervise the patient. The user identification and location system in conjunction with the alert system has helped to notably reduce the percentage of incidents in the environment under study. Moreover, in addition to a reduction in the number of incidents, the time elapsed between the generation 
of a warning and solution decreased significantly. Finally, due to the many improvements, the level of user satisfaction increased with the introduction of HoCa architecture since patients can live in their own homes with the same level of care as those offered at the residence.

\section{References}

1. Ardissono L, Petrone G, Segnan M (2004). A conversational approach to the interaction with Web Services. Computational Intelligence, Blackwell Publishing, 20:693-709.

2. Bahadori S, Cesta A, Grisetti G, Iocchi L, Leone R, Nardi D, Oddi A, Pecora F, Rasconi R (2003). RoboCare: Pervasive Intelligence for the Domestic Care of the Elderly. Artificial Intelligence, 1(1):16-21.

3. Bonino da Silva LO, Ramparany F, Dockhorn P, Vink P, Etter R, Broens T (2007). A Service Architecture for Context Awareness and Reaction Provisioning. In: IEEE Congress on Services (Services 2007). pp. 25-32.

4. Carrascosa C, Bajo J, Julian V, Corchado JM, Botti V (2008). Hybrid multiagent architecture as a real-time problem-solving model. Expert Systems With Applications, 34(1):2-17.

5. Corchado JM, Bajo J, de Paz Y, Tapia D (2008). Intelligent Environment for Monitoring Alzheimer Patients, Agent Technology for Health Care. Decision Support Systems, 34(2):382-396.

6. Corchado JM, Bajo J, Abraham A (2008). GERAmI: Improving the delivery of health care. IEEE Intelligent Systems, 23(2):19-25.

7. ITAA. (2004). Radio Frequency Identification. RFID...coming of age. In White paper, Information Technology Association of America. http://www.itaa.org/rfid/docs/rfid.pdf.

8. Julian V, Botti V (2004). Developing real-time multi-agent systems. Integrated Computer-Aided Engineering, 11(2): 135-149.

9. Mengual L, Bobadilla J, Trivio G (2004). A fuzzy multi-agent system for secure remote control of a mobile guard robot. In: Second International Atlantic Web Intelligence Conference, AWIC 2004, LNCS 3034, pp. 44-53.

10. Ricci A, Buda C, Zaghini N (2007). An agent-oriented programming model for SOA and web services. In: 5th IEEE International Conference on Industrial Informatics, pp. 1059-1064.

11. Rigole P, Holvoet T, Berbers Y (2002).Using Jini to integrate home automation in a distributed software-system. In: 4th International workshop on distributed communities in the Web, LNCS 2468, pp. 291-304.

12. Tapia DI, Bajo J, De Paz F, Corchado JM (2006). Hybrid Multiagent System for Alzheimer Health Care. In: Hybrid Artificial Intelligence Systems. HAIS 2006. Javier Bajo, Emilio S. Corchado, lvaro Herrero and Juan M. Corchado (Eds.). Universidad de Salamanca, pp. 1-18.

13. Walton, C. (2006) Agency and the Semantic Web. Oxford University Press, Inc.

14. Wooldridge, M. (2002). An Introduction to MultiAgent Systems. John Wiley and Sons. Chichester, England.

15. Zhiqun Chen (2000). Java Card Technology for Smart Cards. Addison Wesley Longman. ISBN 0201703297. 